\title{
Definición de los Requerimientos de Información y Funciones para la Gestión de Mantenimiento Mediante un Proceso de Análisis Constructivo
}

\author{
Fernando F. Espinosa y Gonzalo E. Salinas \\ Facultad de Ingeniería, Universidad de Talca, 2 Norte 685, Talca, Chile (e-mail: fespinos@utalca.cl; \\ gsalinas@utalca.cl)
}

Recibido May. 28, 2015; Aceptado Jul. 9, 2015; Versión final Jul. 27, 2015, Publicado Dic. 2015

\begin{abstract}
Resumen
En este trabajo se presenta un procedimiento para formalizar los requerimientos de información que apoyen el proceso de toma de decisiones para una empresa de tamaño mediano del rubro maderero. El procedimiento se basa en los principios de la Metodología Multicriterio de Apoyo a la DecisiónConstructivista para capturar, desde los involucrados con el proceso de mantenimiento, la falta de información, ordenarla y jerarquizarla y de los principios de la Teoría de Sistemas para definir las funciones y los grupos de información. Como un resultado anexo de la aplicación de este procedimiento se obtuvieron las bases para un sistema administrativo. Esto se logra al definir la malla de información, los procedimientos para ejecutar cada función y la estructura de datos para cada grupo de información.
\end{abstract}

Palabras clave: gestión del mantenimiento, MCDA constructivista, sistemas de información, estructura jerárquica.

\section{Definition of the Information Requirements and Functions for Maintenance Management through a Process of Constructive Analysis}

\begin{abstract}
This paper presents a procedure to formalize the information requirements to support the decision making process for a medium-size productive timber plant. The procedure is based on the principles of Constructivist Multicriteria Decision Aid Approach to capture, from those are involved with the process of maintenance, the lack of information, to organize and obtain the hierarchical structure, and from the principles of systems theory to define the functions and the information groups. As an additional result of this procedure the basis for an administrative system was obtained. This is obtained by defining the information network, the procedures to execute each function and the data structure for each group of information.
\end{abstract}




\section{INTRODUCCIÓN}

Generalmente en el segmento de las PYMES, una de las situaciones más recurrentes en el proceso de mejoramiento de su gestión es que cuando necesitan tomar decisiones con una incertidumbre baja, para disminuir costos, mejorar eficiencia, proponer estrategias u otro aspecto inherente al buen desempeño del negocio, se encuentran que la información pertinente, actualizada y completa es un insumo de calidad del cual carecen, o bien usan un sistema de información de uso general que tampoco les entrega la real información que necesitan (Sherwin, 2000; Moore y Starr, 2006; Crespo y Gupta, 2006; Mei-yu et al., 2008). Las verdaderas decisiones gerenciales, por la definición propia de "gestión", requieren juicios personales, es decir, conjeturas sustentadas por la experiencia y complementadas por cálculos parciales basados en datos reales y conocimientos atingentes.

No se trata que las decisiones propias del administrador deban ser automatizadas, sino que se deben tomar con mayor rapidez y ojalá muy cerca del óptimo fijado para ese aspecto crítico de la gestión. Por lo tanto debe tener una mejor información proporcionada por un adecuado sistema de información integrado, capaz de sustentar respuestas mediante los resultados del cálculo de las alternativas previamente definidas en base al proceso de toma de decisiones particular de esa organización. Debido a que un equipo computacional no puede hacer juicios, sino sólo cálculos, los datos de entrada deben ser muy detallados y el modelo más elaborado para que las respuestas sean suficientemente precisas para elegir racionalmente entre las políticas alternativas. Los beneficios de una avanzada tecnología de fabricación no pueden alcanzarse plenamente sin un sistema de información que permita a los tomadores de decisiones, incluidas las decisiones sobre el mantenimiento, calcular rápidamente los requerimientos, analizar los indicadores de eficiencia y hacer proyecciones a través de modelos realistas a partir de datos confiables.

Tsang (2002) identificó cuatro dimensiones estratégicas del mantenimiento donde la primera dimensión es la estrategia de prestación de servicios, refiriéndose a la decisión sobre la externalización frente al mantenimiento realizado con recursos propios. La segunda dimensión de la gestión de mantenimiento estratégico se refiere a la organización y estructura del trabajo. La tercera dimensión de la gestión de mantenimiento estratégico es la concepción del mantenimiento seleccionada para atender el negocio de la empresa y la cuarta dimensión estratégica de la gestión de mantenimiento es la selección del sistema de apoyo que incluye el sistema de información, la capacitación y la gestión del rendimiento y el sistema de recompensas. Cada elemento tiene que ser cuidadosamente seleccionado para apoyar el objetivo general de la organización.

Los sistemas ERP (Enterprise Resources Planning), están ganando terreno en las grandes organizaciones y en cierta medida en las organizaciones de tamaño medio. El poder de las ERP radica en su capacidad de integrar diferentes áreas funcionales dentro de la organización, que es un requisito esencial para la planificación y programación del mantenimiento. La implementación exitosa del sistema requiere una cuidadosa selección del sistema y una estrategia de implementación que es focalizada en la persona (AlTurki, 2011; Maruta 2011). La aplicación de la tecnología de la información más reciente no va a hacer maravillas en una empresa donde los colaboradores no saben cómo hacer uso de las facilidades que la tecnología entrega, o cuando los objetivos de la función mantenimiento dentro de la empresa no se ajustan a la filosofía de las tecnologías de la información (TI) en aplicación (Kans, 2008). Las TI es sólo la tecnología gestionada por sus usuarios. El usuario de la tecnología no tendrá ninguna necesidad de esta si él no tiene un propósito, el cual debe estar en concordancia con los objetivos para la utilización de las TI. Si el objetivo y el propósito son claros, el uso real del sistema de TI podría ser efectivo.

El éxito de un sistema de información se basa en seis aspectos: la calidad del sistema, calidad de la información, el uso del sistema, la satisfacción del usuario, los impactos individuales y los impactos en la organización (Khan y Darrab, 2010). La calidad del sistema, la singularidad, pertinencia y actualización de la información afecta el uso y la satisfacción de los usuarios lo cual está directamente relacionado con la aceptación del sistema. El uso del sistema y la satisfacción del usuario están relacionados entre sí: el uso afecta a la satisfacción de los usuarios positiva o negativamente y viceversa. Estos, a su vez, son antecedentes del impacto en el desempeño individual que a su vez tendrá repercusiones organizativas

Una de las principales causas de los incidentes relacionados con el mantenimiento es la falta de comunicación y transferencia de información dentro y entre las diferentes fases y actividades del proceso de mantenimiento. La falta de información entre los diferentes niveles de mando en la función mantenimiento es también una de las principales causas de eventos "sin encontrar la falla" dentro de industrias, donde los costos, la seguridad y la fiabilidad son afectados negativamente (Karim et al. 2009). Una razón de la importancia de la información pertinente y la comunicación adecuada dentro del mantenimiento es la diversidad de los datos necesarios con el fin de realizar diferentes actividades dentro del proceso de mantenimiento como ser los datos estáticos y dinámicos relacionados con el sistema técnico, recursos y 
organización. Toda la complejidad inherente a la gestión del mantenimiento hace que las decisiones sobre programación y uso de recursos sea más difícil de llevar a cabo por tanto se necesita de variados requerimientos de capacidad de procesamiento de la información de la organización para apoyar un proceso eficiente de tomar decisiones (Swanson, 2003).

\section{EL SISTEMA DE INFORMACIÓN ADECUADO}

Variados factores son los que están presionando por la necesidad de tener un sistema de información para ayudar a la gestión del mantenimiento. Primero, la cantidad de información disponible, aún para la más pequeña organización, está continuamente creciendo. Además, hay un aumento en los requerimientos para tener estos datos e información a mano en tiempo real para la toma de decisiones. En seguida, el tiempo de vida de los datos está disminuyendo como el resultado de la realidad del piso de fábrica, los cuales son en tiempo real por naturaleza, y de rápida tasa de cambio. La tendencia ahora es adquirir los datos sobre las máquinas individualmente, basado sobre interacciones individuales más bien que deducir el comportamiento desde datos históricos. Finalmente la manera como los datos están siendo accedidos ha cambiado, ahora son tablas dinámicas, preguntas en línea que deben ser respondidas en segundos (Labib, 2004).

Esta brecha de apoyo a la toma de decisión es definitivamente un problema, porque la llave para un mantenimiento sistemático y efectivo es gestionar para que esta sea apropiada a las circunstancias particulares de la máquina, planta u organización. Este proceso de toma de decisiones se dificulta si el sistema computacional solamente puede ofrecer un análisis de los datos guardados. Un paso hacia el apoyo a la toma de decisiones es variar la periodicidad de una acción de mantenimiento dependiendo de la combinación de la frecuencia de la falla y la severidad.

Para el diseño adecuado del sistema de información, bajo las condiciones descritas anteriormente, los requerimientos deben nacer desde los futuros usuarios del sistema, ya que estos deben ver reflejados sus intereses por información que realmente les ayude en su proceso de toma de decisiones. La adaptación a la organización de los sistemas de información es un aspecto importante del proceso de desarrollo de la aplicación donde los requerimientos de los profesionales desempeñan un papel clave en el proceso de adaptación (Vasconcelos, 2007), no solamente por lo que refleja en la conformación de los diferentes puntos de vista sobre el papel en la organización de los sistemas de información hecho que no se ve reflejado cuando el sistema es impuesto como estándar para el manejo de datos, aunque el sistema sea uno de los tantos ya existentes. En el proceso de elaboración del sistema de información se debe dar énfasis al papel constructivo y constitutivo del discurso en general y, en particular, de las prácticas complejas en la organización de los sistemas de información (Vasconcelos, 2007), a la calidad y extensión del conocimiento incluido en este (Yehia et al, 2008), al modelado de la estructura informativa y el agrupamiento de los conceptos en una organización eficaz (Ehsani et al, 2010) y en una proyección de futuro en el proceso de toma de decisiones en las tareas de mejoramiento de las acciones de mantenimiento (Moore y Starr, 2006).

Una de las herramientas que se pueden aplicar con éxito para la captura de los requisitos de un sistema de información, directamente desde el piso de fábrica, es el MCDA Constructivista (Constructivist Multicriteria Decision Aid Approach) que ayuda a organizar y sintetizar la información que ha sido recopilada desde los involucrados directamente con el problema en estudio. Este hecho permite obtener un conjunto de alternativas que podrían ser tratadas de forma dinámica y así obtener antecedentes sobre las necesidades de información, de funciones administrativas o de gestión que deben ser realizadas y la documentación necesaria para recopilar y transmitir datos elaborados, ya que el equipo de análisis puede organizar la búsqueda de las soluciones que optimicen la probabilidad de éxito, el tiempo y el costo con los cuales se consigue una solución que aporta valor a la organización (Espinosa y Salinas, 2013).

En lo referente al manejo de la información en el contexto de análisis de decisión multicriterio (MCDA) las aplicaciones más recientes se han realizado en investigar el potencial y los beneficios de la "ayuda inteligente del usuario" con su conocimiento y experiencia en el contexto de uso del sistema de información (Hodgkin et al., 2005), mediante el estudio de las similitudes y diferencias de los requerimientos con sus características distintivas aplicar la metodología a una nueva problemática que es la evaluación multicriterio de la robustez del diseño (Hites et al. 2006), en la ingeniería clínica, para ayudar a la identificación de las dificultades encontradas por los actores relevantes en la toma de decisiones de un proceso tecnológico de la salud tratando de concentrarse en lo que es relevante para la mejora de procesos (De Moraes et al., 2010), en el desarrollo de un enfoque (metodología) que ayuda a gestionar las relaciones de colaboración mediante la identificación y medición, bajo un enfoque integral, tanto de los factores y los elementos de desempeño entre las empresas, teniendo en cuenta sus relaciones recíprocas e internas (Verdecho et al., 2012) y dar una recomendación de cómo tomar una nueva decisión en circunstancias específicas donde MCDA es de ayuda esencial en la estructuración del problema de decisión, análisis de las preferencias del 
decisor, el desarrollo de un modelo integral de una situación de decisión, y su posterior explotación dando lugar a una recomendación (Kadzinski et al., 2010).

\section{CONFORMACIÓN DE LOS MAPAS MEDIO - FIN}

El objetivo de la metodología MCDA es crear las bases para auxiliar en la toma de decisión, para un determinado problema, según la perspectiva de los decisores, ya sean los responsables por la solución del problema, o bien, los principales afectados por el problema en cuestión. Este caso se desarrolló para el área del mantenimiento de una empresa maderera que actúa principalmente bajo el régimen de acciones correctivas, y su aspiración es escalar en la gestión del mantenimiento para que esta sea más efectiva y apoye realmente sus expectativas de un aumento en los niveles de producción y una reducción en los costos, pero su gran problema es la falta de información para proponer y evaluar estrategias de mejoramiento.

El procedimiento que se usó en esta aplicación es descrito en la Fig. 1 y está compuesto de tres grandes grupos de actividades: identificación del contexto de las decisiones, estructuración del problema y finalmente el análisis de la estructura del sistema de información. En la fase de estructuración, es donde se aplica más en profundidad la metodología MCDA y es aquí donde se recopilan en primera instancia los elementos que deberán ser apoyados por el sistema de información, y posteriormente en base a qué elementos más detallados se consigue estructurar el proceso de toma de decisiones, tal que éste represente las perspectivas de los involucrados con el problema. El primer paso es caracterizar el contexto de la decisión, definiendo el contorno del problema, o sea, sus relaciones internas y límites y las personas afectadas. La definición del contexto decisorio va a identificar a los actores involucrados directa y/o indirectamente en el proceso y esos actores son personas o grupos que entregarán las acciones, valores 0 alternativas en la construcción del modelo multicriterio (Espinosa y Salinas, 2013)

En base al contexto decisorio se define un rótulo para el problema y este tiene como objetivo orientar la identificación de los elementos primarios de evaluación (EPEs), delimitando este contexto. Los EPEs representan objetivos, metas, valores y opciones de los decisores los cuales son utilizados como base para la construcción de los mapas de medio - fin. A partir de los EPEs son construidos los conceptos para llevarlos a la acción, que es la parte central de la segunda fase de la metodología.

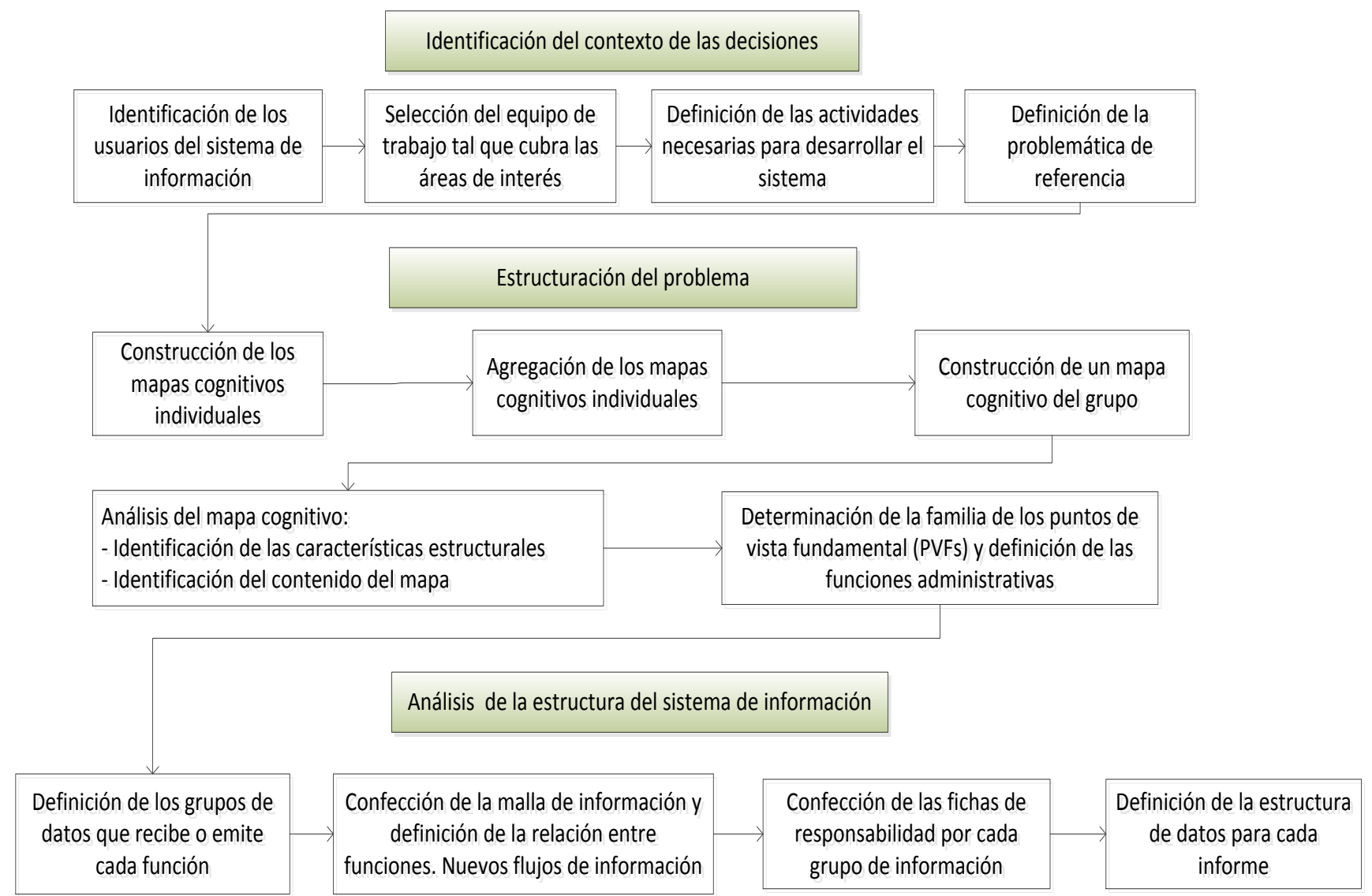

Fig. 1: Procedimiento para el desarrollo del sistema de información 
La obtención de los EPEs se realizó por medio de entrevistas a los encargados del mantenimiento de la empresa maderera, los que fueron guiados por un facilitador, quien en un principio tuvo que realizar una auditoría que contenía aquellos aspectos que en general son los más sensibles para quienes realizan el trabajo cotidiano del mantenimiento, y de esta forma facilitar la tarea y organizar su trabajo tratando de abarcar lo más posible el amplio espectro de posibilidades que el tema entrega. El rótulo y los EPEs indicados por los mantenedores se resumen en la Fig. 2.

Conforme al procedimiento indicado en la Fig. 1 corresponde la realización de los mapas cognitivos agregados del grupo. En esta etapa los participantes siguen explorando sus necesidades ya sea tanto de información como de acciones que deben realizarse para la recopilación de datos desde el piso de fábrica, los cuales se agrupan conforme su naturaleza en puntos de vista fundamentales. Los mapas ordenados se agruparon en dos puntos de vista: aspectos gerenciales y aspectos financieros. En la Fig. 3 se muestran los aspectos gerenciales.

Estos mapas son la materia prima para definir las funciones (actividades administrativas o de gestión asociadas a la toma de decisiones), los flujos de información (conjunto de datos ordenado que ligan las funciones), las acciones (procesos que se realizan producto de una decisión en alguna de las funciones) y el contenido de la base de datos, la cual se podría manifestar como archivos digitales y documentos en el interior del sistema de información con una relación de atributos a veces difícil de percibir (Gento, 2004), pero lo concreto es que el sistema de información es usado para representar el conocimiento de la organización y como apoyo para la gestión de esta.

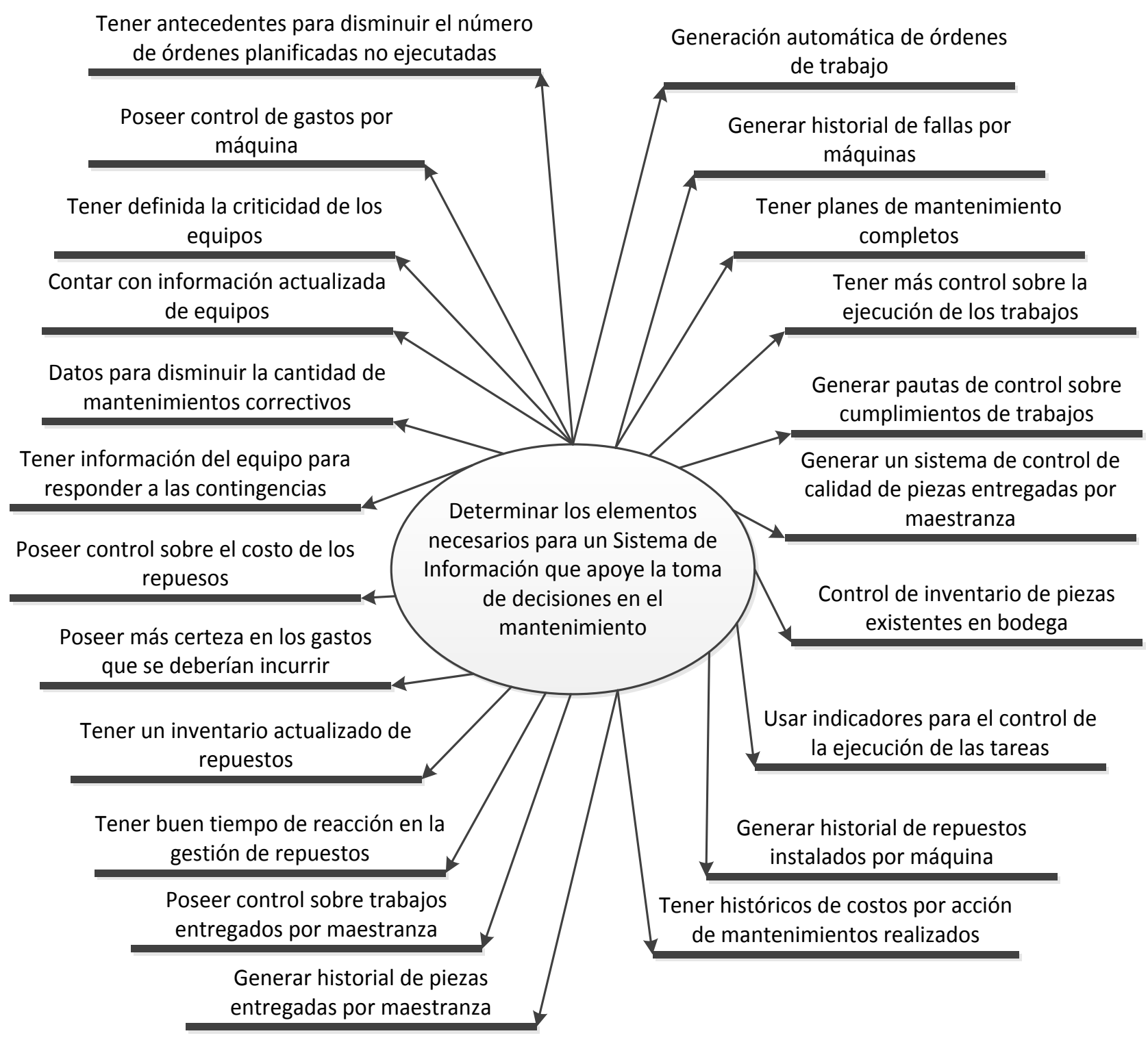

Fig. 2: Rótulo del problema y elementos primarios de evaluación 


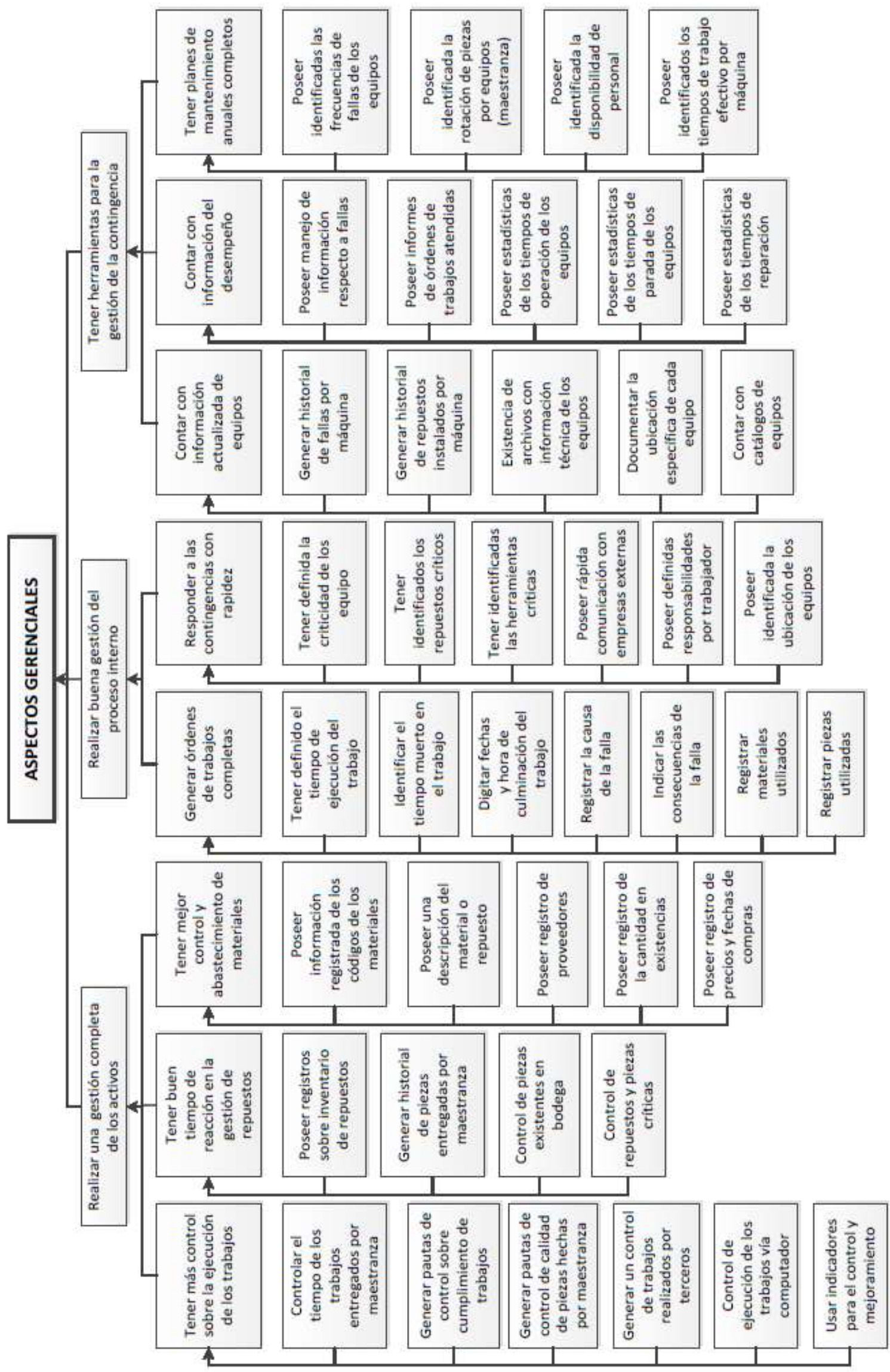

Fig. 3: Mapa medio-fin para los aspectos gerenciales deseados para el sistema de información 


\section{DESARROLLO DEL SISTEMA DE INFORMACIÓN}

Usar un tipo específico de modelado es en general un problema difícil debido a su naturaleza inherentemente subjetiva. Así pues, el modelo adoptado debe habilitar la comunicación natural con el usuario final para garantizar una captura precisa y coherente de sus preferencias (Yeganeh N. et al. 2014, Sun y Teng, 2012). Para el sistema de información de apoyo en el proceso de toma de decisiones en el mantenimiento este debe considerar los siguientes aspectos: a) el contexto general en que existe, b) la función global específica que da origen al sistema bajo diseño, c) las grandes entradas y salidas a la función, d) otras funciones relacionadas con las cuales se intercambian entradas y salidas, y e) los procesos afectados por la función.

En la tercera parte del desarrollo se debe dar la estructura jerárquica de funciones e información, ya que en los pasos anteriores capturados por los mapas medio - fin, los participantes y futuros usuarios del sistema solamente reflejaban sus necesidades de información y acciones indistintamente sin distinguirlas una de otras. La distinción entre funciones (que involucra acciones generales) e información (que son datos necesarios para la decisión) se hace a partir de los mapas ya ordenados solicitando a los participantes que describan lo solicitado en cada EPEs y ellos mismos definan si es función o información. En general para aquellos EPEs que comienzan con un verbo son candidatas a función y entre todas las factibles cuales son de primer nivel, o sea, cuales son más amplias en su definición. En la Fig. 4 se muestra la descomposición en funciones y grupos de información para el aspecto gerencial y en la Fig. 5 la malla de información para todo el sistema.

Contando con capacidad de computación y disponibilidad de información que sea pertinente, cada vez es más importante tener nuevas y más eficientes formas de agrupación de datos para los análisis que apoyen el proceso de toma de decisiones. El almacenamiento y la recuperación de datos se convertirán en grandes temas al interior de la función administrativa, ya que requieren recursos especiales sean estos físicos o humanos. Es importante determinar cómo se utilizarán los datos, y luego establecer una jerarquía para los diversos parámetros de análisis de las condiciones que surgen a partir de la implementación de estrategias de mejoramiento.

En el desarrollo del sistema de información el énfasis debe ser puesto en los resultados, no en las actividades. Para aprovechar realmente la inversión en tecnologías de la información se requiere modelar, e idealmente llegar a la automatización de los análisis con sistemas basados en reglas de expertos. Este hecho de pensar en los resultados es relevante para el desarrollo porque independiza al diseño de las personas, en su comienzo, ya que obliga a los participantes a pensar en lo que falta como información relevante y no en la forma nueva de trabajar, que les puede complicar, aun sabiendo que más delante se deberá definir también ese aspecto.

Un sistema de información actualizado y pertinente ofrece mejores alternativas para una mejor comunicación entre los trabajadores de la planta y expertos externos, y proporciona el vehículo para compartir información, conocimientos, experiencias y el saber hacer. Poseer esta facilidad da ventaja a aquellas empresas cuyo fin es permanecer vigentes en un mercado altamente competitivo ya que este exige producción predecible y confiable. La previsibilidad se ha convertido virtud destacada, y es un claro indicador de la capacidad de gestión para controlar la producción de la planta. Este factor es la razón por la que muchas empresas se han preocupado por mejorar la confiabilidad de los equipos de producción, y por qué las tecnologías más recientes se han centrado en la predicción temprana de las fallas en los equipos.

Vinculando los objetivos del negocio con el del sistema de información ayuda a los mantenedores de las plantas a entender y mejorar la confiabilidad de los equipos. El vínculo no se define por la cantidad de datos que son recogidos y almacenados, sino en cómo los analistas usan eficientemente la información para tomar decisiones que afectan a la disponibilidad. Las mejoras en la productividad de los equipos de producción se centran en los cambios mediante una mejor disponibilidad de información. Si los mantenedores y operadores siguen realizando sus actividades como siempre sin ningún compromiso con el uso de la información para modificar sus prácticas, la inversión en el nuevo sistema se desperdiciara, y es aquí donde esta metodología ayuda a generar ese compromiso porque los requerimientos nacen desde quienes deciden y ejecutan el mantenimiento.

Muchas prácticas existentes son a menudo ineficaces en la identificación y corrección de problemas de los equipos lo que se traducen en falta de disponibilidad de estos. La razón de este hecho se encuentra en la falta de preocupación en la recopilación de los datos, así los sistemas de información existentes no proporcionaran suficiente calidad de análisis, o incluso no proporcionan un acceso adecuado a los datos necesarios para entender la confiabilidad del equipo. 


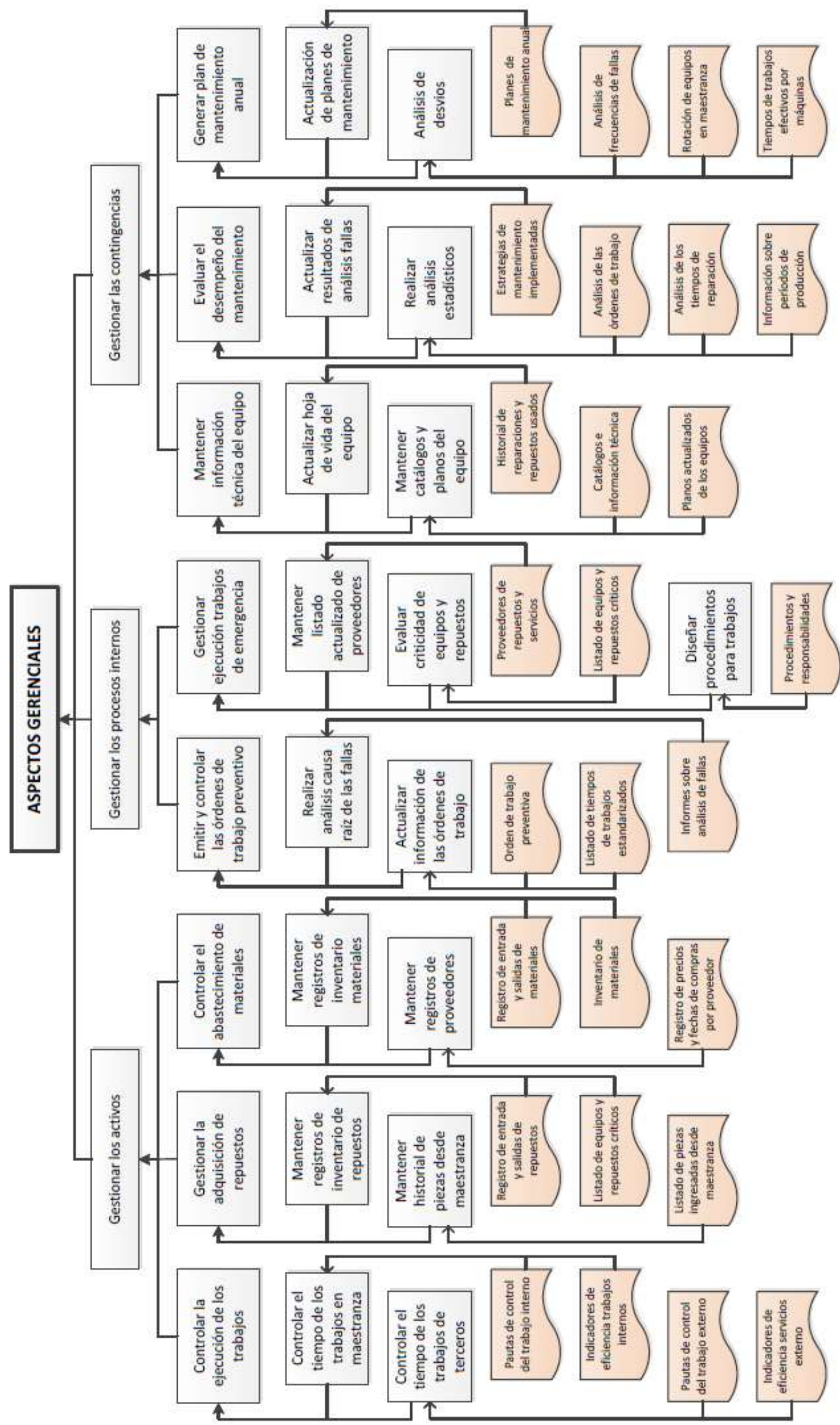

Fig. 4: Mapa medio-fin para los aspectos gerenciales jerarquizados y ordenados para el sistema de información 
Un enfoque optimizado para el mantenimiento incluye una revisión de la información de diagnóstico una vez terminada la reparación. En la mayoría de los casos, este análisis no se realiza debido a la falta de énfasis en los problemas de mantenimiento y hay variadas razones para no llevar a cabo esta revisión, entre las cuales se puede mencionar la falta de herramientas para la captura de datos y la preparación para analizar estos datos, la falta de procedimientos que muchas veces lleva a relevar las responsabilidades de cada cargo dentro de la organización, y la falta de comunicación entre los operadores y mantenedores que muchas veces es a causa de la diferencia en la comprensión de sistemas tanto productivos como administrativos. Para minimizar los efectos negativos mencionados en los párrafos anteriores el siguiente paso en el desarrollo es construir la malla de información del sistema administrativo donde se especifique el origen y destino de cada grupo de información que se detallaron en los mapas medio - fin. El objetivo es definir la dinámica del sistema de información, o sea, para cada función de donde proviene o envía la información que maneja, con que finalidad y cuál es el proceso de transformación a que es sometida. También se refina la cantidad de grupos de información generando nuevos grupos que principalmente cumplen la misión de crear bucles de control. La malla de información es mostrada en la Fig. 5.

Además en el proceso de confección de la malla de información, naturalmente nace la descripción del procedimiento que debe atender cada función para que el flujo de información sea relevante. Este procedimiento posteriormente indicará la responsabilidad de la persona que asuma la ejecución de las tareas que conlleva cada función. Este procedimiento consensuado entre los participantes del proyecto se vacía en una ficha para cada función y en la Fig. 6 se muestra una parte del documento para la función Emitir y Controlar las Ordenes de Trabajo Preventivo a modo de ejemplo.

El último paso del procedimiento dice relación con la estructura de datos que debe contener cada grupo de información. También esto se realizó en base a un trabajo colaborativo y la pregunta inicial fue: para que este flujo de información (en la mayoría de los casos se convirtió en un documento) sea operativo ¿qué datos específicos se necesitan? Nuevamente se aplica el criterio de jerarquizar los datos y preguntarse si realmente es necesario tenerlo para ejecutar la acción definida en la función, si no está comprendido en otro y cómo aporta al resultado de tener dicho informe. La idea central es que se rescaten solamente aquellos datos estrictamente necesarios para no entrampar el proceso de elaboración o análisis de información. En la Fig. 7 se muestra una parte del resultado para una Orden de Trabajo. Hay que tener presente que los mantenedores y los ingenieros necesitan tener acceso a más y más datos pertinente para tomar decisiones sobre estrategias de mejoramiento con alta eficacia, en especial información con énfasis en la disponibilidad, impulsada por la competencia en el tiempo de producción de la línea. Mejora en la disponibilidad significa menos tiempo en un estado de detención del equipo productivo.

Una de las grandes ventajas que ofrece la aplicación del MCDA es que involucra a los usuarios junto a los facilitadores en el diseño del sistema bajo estudio y eso es importante en las siguientes fases del proyecto. Como la metodología es muy demandante de tiempo de los colaboradores toma especial importancia la figura del facilitador para mantener la dinámica del grupo, especialmente presentando resultados de las encuestas y de las reuniones. Una vez que este sistema comience a dar resultados, se organice bien la parte administrativa de la función mantenimiento y se adquiera experticia en el manejo de datos se podrá comenzar con la implementación del apoyo computacional y aprovechar la oportunidad de ingresar los nuevos requerimientos que sin duda aparecerán, como ser indicadores de eficiencia, análisis de tendencias, sistemas expertos, entre otros. Este proyecto es para la empresa solo el comienzo de un camino de mejora continua.

Tal como está planteada la solución para la empresa a su problema de falta de información va a ser relativamente fácil para los encargados del mantenimiento seleccionar o elaborar la herramienta computacional, ya que tienen claridad en el detalle de los datos a recopilar y sólo restaría definir la organización en un documento o pantalla de computador. En un comienzo las expectativas de la empresa se centraban en la falta de información que les impedía cumplir satisfactoriamente sus metas, sin embargo el paso hacia un sistema administrativo organizado fue casi natural ya que preguntas relacionadas con el objetivo de cada flujo de información, quien iba a ser el responsable de cada flujo, quien las usaría en el proceso de toma de decisiones.

\section{CONCLUSIONES}

El análisis realizado en este trabajo permite obtener las siguientes conclusiones principales: i) una de las grandes ventajas que ofrece la aplicación del MCDA es que involucra a los usuarios junto a los facilitadores en el diseño del sistema bajo estudio: ii) el procedimiento aumenta la coordinación entre personas ya que obliga a trabajar en equipos de discusión sobre la estructura de los grupos de información, y iii) el proyecto entrega las bases para que la empresa comience un camino de mejora continua. 


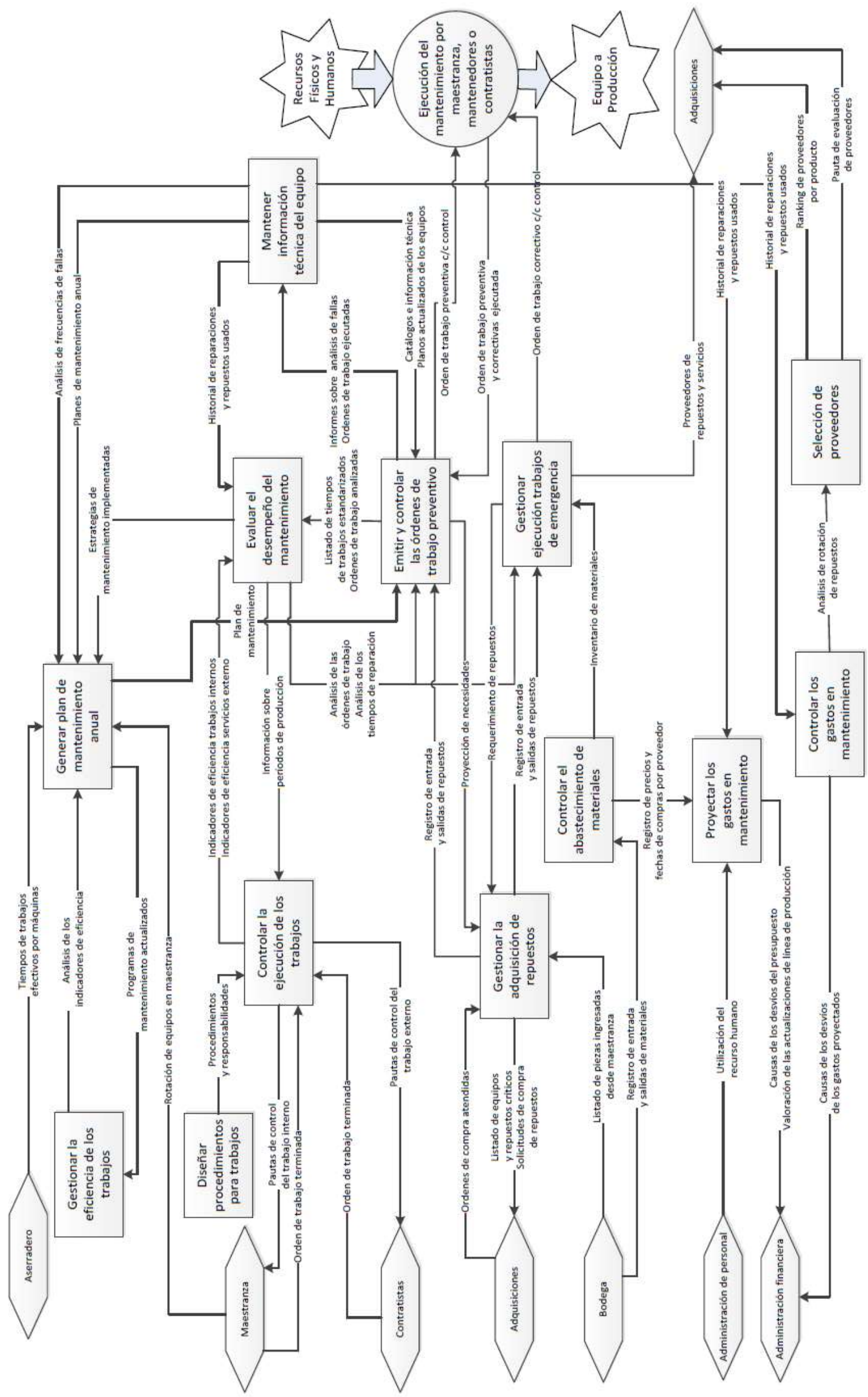

Fig. 5: Malla de información para la función global gestión del mantenimiento 


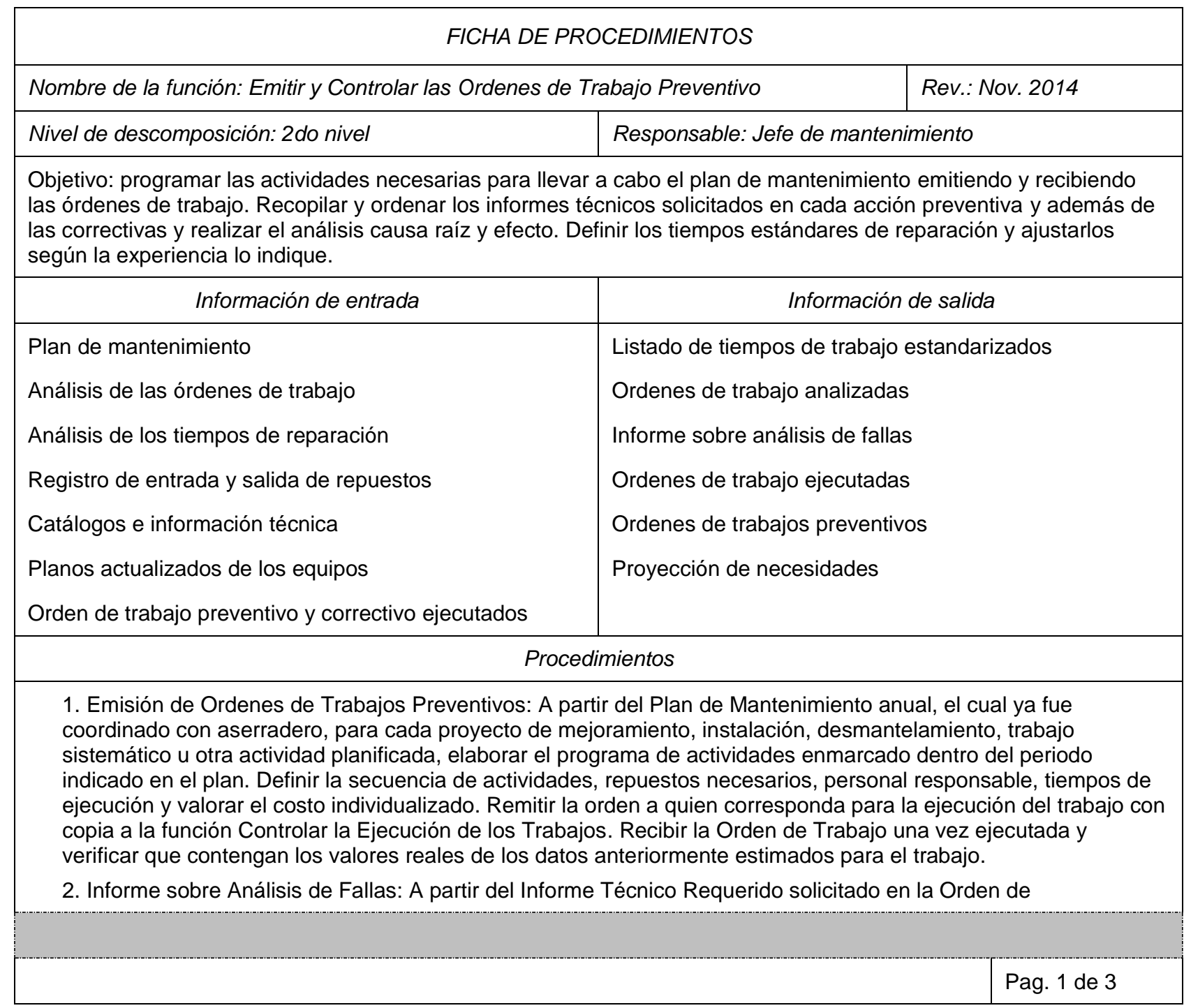

Fig. 6: Ficha de procedimientos para la función Emitir y Controlar las Ordenes de Trabajo Preventivo

\begin{tabular}{|c|c|}
\hline Documento: Orden de Trabajo Preventivo & Rev.: Nov. 2014 \\
\hline 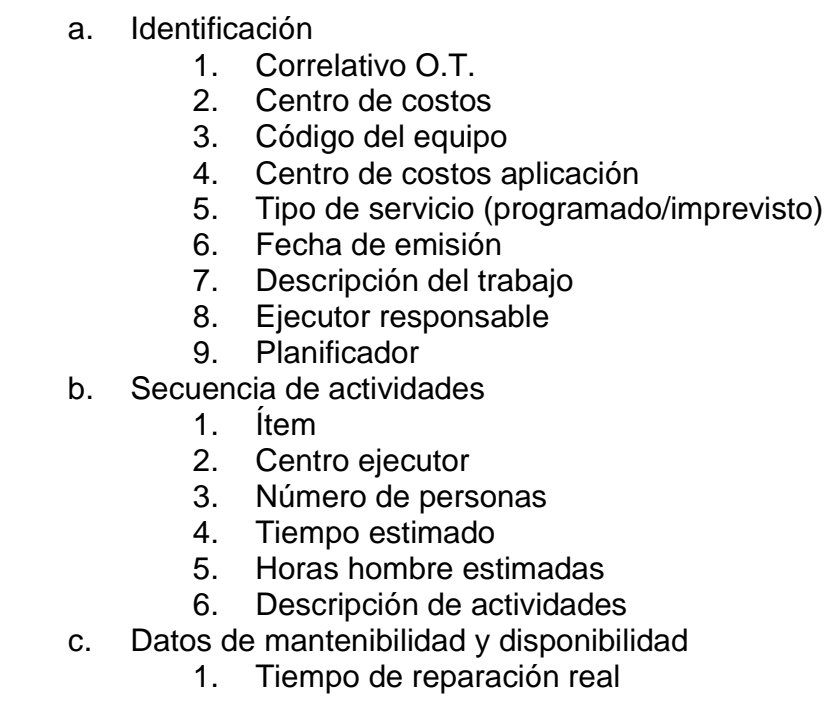 & \\
\hline
\end{tabular}

Fig. 7: Jerarquización de los datos para el flujo Orden de Trabajo 


\section{REFERENCIAS}

Ashraf W. Labib. A decision analysis model for maintenance policy delection using a CMMS. Journal of Quality in Maintenance Engineering. Vol. 10 No. 3, 191-202, (2004)

Crespo A., Gupta J. Contemporary maintenance management: process, framework and supporting pillars. Omega 34, 313 - 326, (2006)

De Moraes L., Garcia R., Ensslin L., Da Conceição M. The multicriteria analysis for construction of benchmarkers to support the Clinical Engineering in the Healthcare Technology Management. European Journal of Operational Research 200, 607-615, (2010)

Ehsani M., Makui A., Nezhad S.S. A methodology for analyzing decision networks, based on information theory. European Journal of Operational Research 202, 853-863, (2010)

Espinosa F., Salinas G. Selección de Estrategias de Mejoramiento de las Condiciones de Trabajo para la Función Mantenimiento Utilizando la Metodología MCDA Constructivista. Inf. Tecnol. 24(3), 57-72, (2013)

Gento A. Decision rules for a maintenance database. Journal of Quality in Maintenance Engineering. Volume 10, Number 3, 210-220, (2004)

Hites R., De Smet Y., Risse N., Salazar-Neumann M., Vincke P. About the applicability of MCDA to some robustness problems. European Journal of Operational Research Volume 174, Issue 1, 1, 322-332, (2006)

Hodgkin J., Belton V., Koulouri A. Supporting the intelligent MCDA user: A case study in multi-person multicriteria decision support. European J. Operational Research, Volume 160, Issue 1, 1, 172-189, (2005)

Kadzinski M., Greco S., Słowinski R. Selection of a representative value function in robust multiple criteria ranking and choice. European Journal of Operational Research 217, 541-553, (2012)

Laura Swanson. An information-processing model of maintenance management. Int. J. Production Economics 83, 45-64, (2003)

M.R. Rotab Khan and Ibrahim A. Darrab. Development of analytical relation between maintenance, quality and productivity. Journal of Quality in Maintenance Engineering. Vol. 16 No. 4, 341-353, (2010)

Maruta R. Transforming knowledge workers into innovation workers to improve corporate productivity. Knowledge-Based Systems 30, 35-47, (2012)

Mei-yu L., Ke-qi H., Xiao-yong Z., Xiao-lin L., Yong-jun Z., Wei-hu Y. A management information system for mine railway transportation equipment. J China Univ Mining \& Technol 18, 373-376, (2008)

Mirka Kans. An approach for determining the requirements of computerized maintenance management systems. Computers in Industry 59, 32-40, (2008)

Moore W. J., Starr A. G. An intelligent maintenance system for continuous cost-based prioritisation of maintenance activities. Computers in Industry 57, 595-606, (2006)

Ramin Karim, Olov Candell, Peter Söderholm. E-maintenance and information logistics: aspects of content format. Journal of Quality in Maintenance Engineering, Vol. 15 No. 3, 308-324, (2009)

Sherwin, David. Software reliability modeling in distributed development environment. Journal of Quality in Maintenance Engineering Vol.6, № 3, 138-164, (2000)

Sun J., Teng J. Information Systems Use: Construct conceptualization and scale development. Computers in Human Behavior 28, 1564-1574, (2012)

Tsang, A.H.C. Strategic dimensions of maintenance management, Journal of Quality in Maintenance Engineering, Vol. 8 No. 1, 7-39, (2002)

Umar Al-Turki. A framework for strategic planning in maintenance. Journal of Quality in Maintenance Engineering Vol. 17 No. 2, 150-162, (2011)

Vasconcelos A., The role of professional discourses in the organizational adaptation of information systems. International Journal of Information Management 27, 279-293, (2007)

Verdecho M-J., Alfaro-Saiz J-J., Rodriguez-Rodriguez R., Ortiz-Bas A. A multi-criteria approach for managing inter-enterprise collaborative relationships. Omega 40, 249-263, (2012)

Yeganeh N., Sadiq S., Sharaf M. A framework for data quality aware query systems. Information Systems 46, 24-44, (2014)

Yehia S., Abudayyeh O., Fazal I., Randolph D. A decision support system for concrete bridge deck maintenance. Advances in Engineering Software 39, 202-210, (2008) 